\title{
Brain stimulation improves memory in autism mouse models
}

\author{
BY CHARLES Q. CHOI
}

15 OCTOBER 2021

Chronic electrical stimulation of the fornix, a bundle of nerve fibers deep in the brain, rescues learning and memory deficits in mice with mutations of the autism-linked gene CDKL5, according to new research.

The results support previous work in mice suggesting that electrical jolts to this fiber tract, which links brain regions involved in memory, could help address cognitive problems in multiple models of neurodevelopmental conditions. These animal studies all use deep brain stimulation (DBS), in which electrodes are placed chronically or, in some cases, permanently in specific neuroanatomical regions.

In people, severe cognitive impairment, including memory and learning deficits, is a central feature of cyclin-dependent kinase-like 5 (CDKL5) deficiency disorder, which results from mutations that impair production of the CDKL5 protein. Other characteristics include autism traits and epileptic seizures.

"Our hope is to help CDKL5 deficiency patients with at least some aspects of their problems - for example, intellectual disability," says lead investigator Jianrong Tang, associate professor of pediatrics at the Baylor College of Medicine in Houston, Texas.

\section{Hitting the hippocampus:}

Little is known about how the loss of CDKL5 affects brain circuitry. In the new study, Tang and his colleagues analyzed the brain's memory center, the hippocampus, in mice with CDKL5 mutations. The connections between neurons there were less flexible, they found, which likely contributed to the animals' deficits in learning and memory. The mutations also strengthened inhibitory signals in the dentate gyrus, a part of the hippocampus that helps form new memories. 


\section{Spectrum | Autism Research News}

https://www.spectrumnews.org

DBS stimulation to the fornix for an hour each day over two weeks blunted the abnormal inhibitory signaling. It also restored the animals' performance on learning and memory tasks: Three weeks after stimulation, treated mice were more likely than untreated ones to remember the circumstances in which they received an unpleasant but harmless electric shock to their feet.

Injecting a drug that blocks neuronal inhibition, called gabazine, directly into the dentate gyrus also improved performance on this task. This suggests pharmaceutical or gene therapies targeting this brain circuit may be worth exploring to treat people, Tang says. The work was published in September in the Journal of Neuroscience.

Previously, Tang and his colleagues found that DBS targeting the fornix could improve learning and memory deficits in a mouse model of Rett syndrome, another condition related to autism.

The fact that stimulating the fornix can address learning and memory deficits in two different mouse models suggests the approach may be applicable to more than one condition in people, says Jacque Pak Kan Ip, assistant professor of biomedical sciences at the Chinese University of Hong Kong, who was not involved in this work. "I think this study is very encouraging."

But before this research can find clinical applications, more questions need answers.

For one, it's unclear whether the kinds of improvements documented in this study endure, says Joe Zhou, professor of genetics at the University of Pennsylvania, who was not involved in the study. Further tests in animal models should address that concern.

Future work should also pinpoint the best timing to apply the stimulation, Ip says. "If it is to be used in adults, would that be too late and limit the effects? Or should it be used as young as possible to maximize the effects?"

If so, scientists need to establish whether DBS, an invasive technique that requires brain surgery, is safe in children and whether it might affect other aspects of development, Ip says.

Cite this article: https://doi.org/10.53053/QDKR1080 